\title{
Uterine Corpus Carcinoma and Carcinosarcoma pN1 mi TNM Finding v8
}

National Cancer Institute

\section{Source}

National Cancer Institute. Uterine Corpus Carcinoma and Carcinosarcoma pN 1 mi TNM

Finding v8. NCI Thesaurus. Code C139781.

Uterine corpus carcinoma or carcinosarcoma with regional lymph node metastasis

(greater than $0.2 \mathrm{~mm}$ but nor greater than $2.0 \mathrm{~mm}$ in diameter) to pelvic lymph nodes.

(from AJCC 8th Ed.) 